\title{
Molecular Species of Phospholipids of Lilium longiflorum Pollen
}

\author{
Jiro Sekiya, Kazuhiko Yamashita and Noboru Shimose \\ Department of Agricultural Sciences, Faculty of Agriculture, \\ Okayama University, Tsushima, Okayama 700, Japan \\ Received August 16, 1989
}

\begin{abstract}
Molecular species of the major phospholipids, phosphatidylcholine (PC, $49 \%$ of total phospholipids), phosphatidylethanolamine (PE, 27\%), and phosphatidylinositol (PI, 11\%), of Lilium longiflorum pollen grains were analyzed. Fatty acid compositions (sn-1/sn-2) of major molecular species of PC, PE, and PI were $18: 3 / 18: 3,18: 2 / 18: 3$ (and/or $18: 3 / 18: 2$ ), 18:2/18:2, 16:0/18:3, and 16:0/18:2. Molecular species compositions were quite similar among PC, PE and PI. Mature pollen grains contained more unsaturated molecular species than immature pollen grains.
\end{abstract}

Pollens of many plant species are resistant to dryness and organic solvents. On pollination, pollens imbibe water on the stigma, germinate, and elongate pollen tubes quickly. During pollination the plasma membrane of pollen grain seems to be important together with the cell wall.

Phospholipids are one of major lipid classes in pollen grains. ${ }^{1)}$ Among phospholipids, PC, $\mathrm{PE}$, and PI are the predominant lipids in pollen grains of Pinus species, ${ }^{1,2)}$ tea, ${ }^{3)}$ rice, ${ }^{4)}$ maize, ${ }^{4)}$ and tobacco. ${ }^{5}$ Fatty acid compositions of these phospholipids have been reported. However, molecular species composition may be more important than fatty acid composition to understand the physiological phenomena in which membranes are involved. For example, the content of saturated molecular species of PG has been proposed to be critical in chilling sensitivity. ${ }^{6}$ )

Recent development of an analytical method for phospholipids enabled us to identify molecular species quickly. ${ }^{7,9)}$ We have attempted to understand the role of phospholipids in pollen membranes and found that the predominant molecular species of tea phospholipids, $\mathrm{PC}$ and $\mathrm{PE}$, are $18: 3 / 18: 3$ and $16: 0 / 18: 3{ }^{3)}$ In this report, we describe the molecular species composition of PC, PE, and PI isolated from Lilium longiflorum pollen grains and a change in molecular species during the late stage of pollen development.

\section{Materials and Methods}

Plant materials. Lily flower (Lilium longiflorum Thumb cv. Hinomoto) were obtained from a local greenhouse. Mature pollen grains were collected from fully opened flowers and immature pollen grains from anthers of flower buds $7-9 \mathrm{~cm}$ long. The germination rate of hexane-washed mature pollen was $50-70 \%$ on a synthetic agar medium. ${ }^{10}$ )

Chemicals. Naja naja venom phospholipase $\mathrm{A}_{2}$, Bacillus cereus phospholipase $\mathrm{C}$, authentic phospholipids (PC, PE, PI, PG, phosphatidylserine, and cardiolipin), and authentic PC $(18: 2 / 18: 2,16: 0 / 18: 2,18: 1 / 18: 1,16: 0 / 18: 1$, $18: 1 / 16: 0,18: 0 / 18: 0,16: 0 / 16: 0)$ were purchased from Sigma Chemical Co., and authentic PC (18:3/18:3) from Avanti Polar-Lipids, Inc. 3,5-Dinitrobenzoylchloride was obtained from Wako Chemical Industries, Ltd. Precoated silica gel TLC plates were products of E. Merck (Silica gel 60 ) and Whatman Inc. (PLK 5). All organic solvents used were glass-distilled.

Isolation of phospholipids. Lipids were prepared essentially by the method of Allen and Good, ${ }^{11}$ after washing pollen grains with hexane to remove pigments from the surface. Extracted lipids were chromatographed on a PLK5 preparative plate with $\mathrm{CHCl}_{3}-\mathrm{MeOH}-\mathrm{H}_{2} \mathrm{O}$ $(65: 25: 4, v / v)$. Each phospholipid band was scraped off

Abbreviations: PC, phosphatidylcholine; PE, phosphatidylethanolamine; PG, phosphatidylglycerol; PI, phosphatidylinositol; $16: 0$, palmitic acid; $18: 0$, stearic acid; $18: 1$, oleic acid; $18: 2$, linoleic acid; $18: 3$, linolenic acid. 
Table I. Fatty Acid Compositions of MAJor Phospholipids Isolated From L. longiflorum POLLEN GRAINS

\begin{tabular}{cccccccc}
\hline & & \multicolumn{5}{c}{ Fatty acid composition $(\%)^{b}$} \\
\cline { 3 - 7 } Lipid class & $\begin{array}{c}\text { Content }^{b} \\
(\mathrm{mg} / \mathrm{g} \text { pollen })\end{array}$ & $16: 0$ & $18: 0$ & $18: 1$ & $18: 2$ & $18: 3$ & Others \\
\hline PC & $12.3 \pm 1.2(49)^{c}$ & $43.3 \pm 3.7$ & $1.8 \pm 0.2$ & $4.2 \pm 0.1$ & $24.5 \pm 1.7$ & $24.6 \pm 2.1$ & 0.8 \\
PE & $6.7 \pm 0.8(27)$ & $37.9 \pm 2.1$ & $1.2 \pm 0.2$ & $2.8 \pm 0.6$ & $25.9 \pm 1.6$ & $29.9 \pm 2.6$ & 2.3 \\
PI & $2.8 \pm 0.5(11)$ & $48.3 \pm 4.9$ & $1.2 \pm 0.2$ & $2.1 \pm 0.2$ & $20.3 \pm 2.4$ & $27.0 \pm 2.4$ & 1.1 \\
\hline
\end{tabular}

a Calculated from phosphorus content. Values are means \pm S.E. $(n=3)$

$b \quad$ Values are means \pm S.E. $(n=5)$.

c Values in parentheses represent relative values in $\%$ to total phospholipids.

after visualization with $\mathrm{I}_{2}$, vapor, extracted with $\mathrm{CHCl}_{3}-$ $\mathrm{MeOH}(2: 1, v / v)$, and concentrated in vacuo. Each phospholipid fraction thus obtained was again chromatographed on a TLC plate (PLK5) with $\mathrm{CHCl}_{3}-\mathrm{MeOH}-$ $\mathrm{NH}_{4} \mathrm{OH}(65: 35: 5, \mathrm{v} / \mathrm{v})$. Phospholipids visualized with $\mathrm{I}_{2}$ were scraped off and extracted with $\mathrm{CHCl}_{3}-\mathrm{MeOH}(2: 1$, $\mathrm{v} / \mathrm{v})$. Phospholipids obtained were confirmed both by comparison of $R f$ values with those of authentic lipids and coloring reactions with specific reagents after twodimensional development. ${ }^{31}$

Analytical methods for phospholipids. Other analytical methods for two-dimensional TLC, fatty acid analysis, measurement of phosphorus, phospholipase $\mathrm{A}_{2}$ treatment, preparation of 3,5-dinitrobenzoyl derivatives of phospholipids, and molecular species analysis with HPLC were substantially the same as those described in our previous. paper. ${ }^{3)}$

\section{Results}

\section{Phospholipid classes}

Mean lipid content in mature pollen grains was $222 \pm 11 \mathrm{mg}$ per gram of hexane-washed pollen $(n=7)$. Of total lipids, $11 \%$ was phospholipids based on phosphorus measurement, assuming an average phospholipid molecular weight of 750 and one phosphorus atom per molecule. Therefore, phospholipids accounted for $2.5 \%$ of the fresh weight of hexane-washed mature pollen. PC $(49 \%), \mathrm{PE}(27 \%)$, and PI $(11 \%)$ were the predominant phospholipid classes (Table I). PG, phosphatidylserine (PS), and some other unidentified phospholipids were detected as a minor lipid class, but these were not further analyzed. Traces of glycolipids, monogalactosyldiacylglycerol (MGDG), digalactosyldiacylglycerol (DGDG),
Table II. Positional Distribution of Fatty ACIDS OF Hydrogenated PC

\begin{tabular}{|c|c|c|c|c|}
\hline \multirow{2}{*}{ Lipid } & \multirow{2}{*}{ Position } & \multicolumn{3}{|c|}{$\begin{array}{l}\text { Fatty acid composition } \\
\qquad(\%)\end{array}$} \\
\hline & & $16: 0$ & $18: 0$ & $\begin{array}{l}18: 1+18: 2 \\
\quad+18: 3\end{array}$ \\
\hline Native PC & Whole & 34 & 2 & 64 \\
\hline \multirow{3}{*}{ Hydrogenated $\mathrm{PC}^{a}$} & Whole & 36 & 63 & 1 \\
\hline & $s n-1$ & 63 & 33 & 4 \\
\hline & $s n-2$ & 9 & 91 & 0 \\
\hline
\end{tabular}

a PC was hydrogenated in the presence of Raney nickel and then treated with phospholipase $\mathrm{A}_{2}$. Fatty acids were analyzed by GLC after methanolysis.

and sulfoquinovosyldiacylglycerol (SQDG), were present.

Fatty acid compositions of $P C, P E$, and $P I$

The major faty acid constituents of $\mathrm{PC}, \mathrm{PE}$, and PI were 16:0, 18:2, and 18:3 (Table I). These fatty acids accounted for more than $90 \%$ of the fatty acid constituents. Hydrogenation of PC followed by phospholipase $\mathrm{A}_{2}$ treatment and measurement of fatty acid composition showed that $88 \%$ of $16: 0$ was present at $s n-1$ of a glycerol moiety and $18: 2$ and $18: 3$ attached to both $s n-1$ and $s n-2$ positions (Table II).

\section{Molecular species of $P C, P E$, and PI}

Molecular species of phospholipids were analyzed by conversion of the lipids to 3,5dinitrobenzoyl derivatives followed by HPLC. 
Table III. Identification of Molecular Species of PC Isolated from L. longiflorum POLLEN GRAINS

\begin{tabular}{|c|c|c|c|c|c|c|c|c|}
\hline \multirow{2}{*}{ Peak no. ${ }^{a}$} & \multirow{2}{*}{$\begin{array}{c}t_{R} \\
(\mathrm{~min})\end{array}$} & \multirow{2}{*}{ Evidence $^{b}$} & \multicolumn{5}{|c|}{ Fatty acid composition $(\%)$} & \multirow{2}{*}{$\begin{array}{c}\text { Fatty acid } \\
s n-1 / s n-2\end{array}$} \\
\hline & & & $16: 0$ & $18: 0$ & $18: 1$ & $18: 2$ & $18: 3$ & \\
\hline 1 & 4.48 & $A, B, C$ & 21 & 2 & 0 & 1 & 76 & $18: 3 / 18: 3$ \\
\hline 2 & 4.60 & n.d. & & & & & & \\
\hline 3 & 5.60 & A & 3 & 1 & 1 & 44 & 51 & $(18: 2 / 18: 3)^{c}$ \\
\hline 4 & 7.11 & A, B & 9 & 2 & 1 & 84 & 4 & $18: 2 / 18: 2$ \\
\hline 5 & 7.39 & n.d. & & & & & & \\
\hline 6 & 7.76 & $A, C$ & 47 & 1 & 1 & 0 & 54 & $16: 0 / 18: 3$ \\
\hline 7 & 9.57 & n.d. & & & & & & \\
\hline 8 & 10.06 & A, B & 40 & 3 & 1 & 42 & 14 & $16: 0 / 18: 2$ \\
\hline 9 & 10.59 & n.d. & & & & & & \\
\hline 10 & 13.11 & n.d. & & & & & & \\
\hline 11 & 13.89 & $\mathrm{~B}$ & & & & & & $16: 0 / 16: 0$ \\
\hline
\end{tabular}

a Peak numbers are the same as those in Fig. 1.

b A, fatty acid analysis; B, comparison of retention time with authentic specimens; C, comparison of retention time with tea pollen lipid ${ }^{3)}$; n.d., not determined because of low content.

Mixture of $18: 2 / 18: 3$ and $18: 3 / 18: 2$.

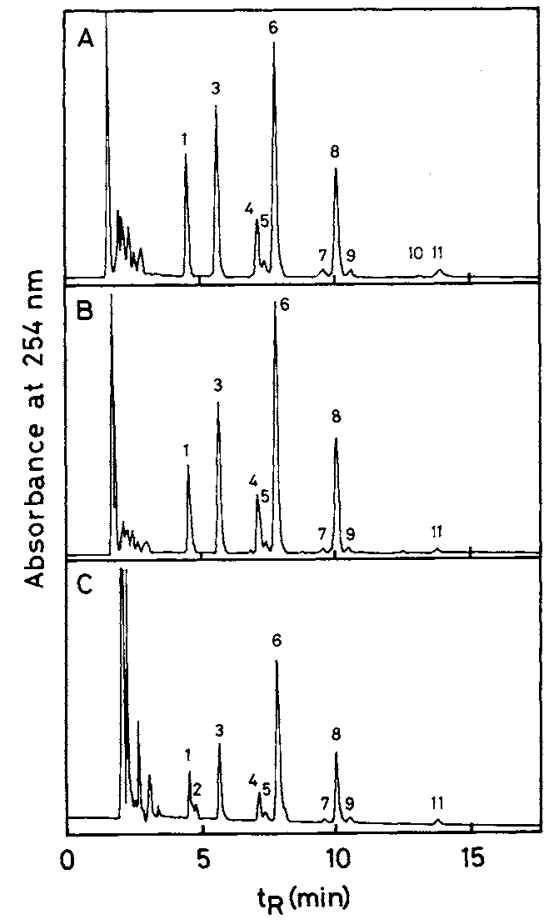

Fig. 1. Typical HPLC Profiles of 3,5-Dinitrobenzoyl Derivatives of PC (A), PE (B), and PI (C) Isolated from Mature Pollen Grains.

Samples of the derivatives were injected into an HPLC mounting Nucleosil $3 C 18$ column $(4.8 \times 150 \mathrm{~mm})$. Mobile phase was acetonitrile-2-propanol $(80: 20, \mathrm{v} / \mathrm{v})$.
Eleven distinct peaks were detected (Fig. 1). Peaks 1, 3, 4, 6, and 8 were the major ones. These 5 peaks accounted for about $90 \%$ of 11 peaks. To identify molecular species of the major peaks, each peak was collected repeatedly and the fatty acid composition was analyzed (Table III). Retention times of the peaks were compared with those of authentic specimens prepared from commercially available phospholipids and those of tea pollen lipids. ${ }^{3)}$ Fatty acid constituents of the peaks 1 , $3,4,6$, and 8 were then measured after methanolysis followed by GLC analysis (Table III). However, the positional specificity of fatty acids in peak 3 could not be confirmed because two isomers were eluted at the same retention time.

The position of 16:0 was found by analysis of hydrogenated $\mathrm{PC}$, which indicates that most of 16:0 attaches to $s n-1$ of glycerol moiety (Table II). This result supports the hypothesis that the peaks 6 and 8 are $16: 0 / 18: 3$ and $16: 0 / 18: 2$, respectively, but not $18: 3 / 16: 0$ and $18: 2 / 16: 0$. Evidence for identification of molecular species of $\mathrm{PC}$ is summarized in Table III.

Major molecular species were all polyunsaturated ones although $38-48 \%$ of fatty acid 
Table IV. Molecular Species Compositions of PC, PE, AND Isolated from Mature L. longiflorum POLLEN GRAINS

\begin{tabular}{rcrrr}
\hline & \multicolumn{5}{c}{ Molecular species composition } \\
$\begin{array}{r}\text { Peak } \\
\text { no. }{ }^{a}\end{array}$ & $\begin{array}{c}\text { Fatty acid } \\
\text { sn-1/sn-2 }\end{array}$ & \multicolumn{3}{c}{$(\%)^{b}$} \\
\cline { 3 - 5 } & & $\mathrm{PC}$ & $\mathrm{PE}$ & $\mathrm{PI}$ \\
\hline 1 & $18: 3 / 18: 3$ & $11.4 \pm 1.7$ & $9.1 \pm 1.0$ & $6.9 \pm 0.5$ \\
2 & - & $0.1 \pm 0.1$ & 0 & $1.3 \pm 0.5$ \\
3 & $(18: 2 / 18: 3)^{c}$ & $17.6 \pm 1.3$ & $16.2 \pm 1.0$ & $10.3 \pm 4.4$ \\
4 & $18: 2 / 18: 2$ & $6.5 \pm 0.4$ & $6.8 \pm 0.7$ & $4.3 \pm 2.2$ \\
5 & - & $2.6 \pm 0.2$ & $2.0 \pm 0.3$ & $1.4 \pm 0.5$ \\
6 & $16: 0 / 18: 3$ & $36.0 \pm 1.4$ & $39.3 \pm 1.6$ & $42.7 \pm 2.8$ \\
7 & - & $1.5 \pm 0.2$ & $1.1 \pm 0.2$ & $0.9 \pm 0.1$ \\
8 & $16: 0 / 18: 2$ & $18.5 \pm 1.2$ & $20.3 \pm 0.4$ & $20.3 \pm 1.6$ \\
9 & - & $1.8 \pm 0.2$ & $1.4 \pm 0.5$ & $1.8 \pm 0.1$ \\
10 & - & $0.2 \pm 0.1$ & $0.1 \pm 0.1$ & $0.3 \pm 0.3$ \\
11 & $16: 0 / 16: 0$ & $1.7 \pm 0.3$ & $1.0 \pm 0.2$ & $0.9 \pm 0.5$ \\
& & & &
\end{tabular}

a Peak numbers are the same as those in Fig. 1.

b Compositions were calculated from peak areas in Fig. 1. Values are means \pm S.E. ( $n=3$ for PC and PE, and $n=2$ for PI),

Mixture of $18: 2 / 18: 3$ and $18: 3 / 18: 2$.

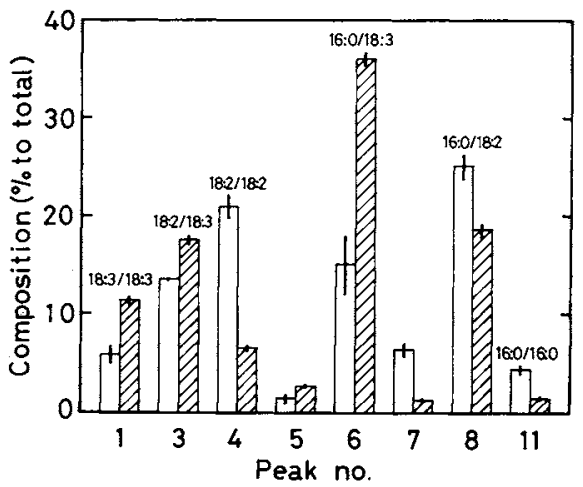

Fig. 2. Changes in Molecular Species Composition of PC Isolated from Immature and Mature Pollen Grains.

Peak numbers are the same as those in Fig. 1. Open bars represent mean \% distributions for immature pollen grains collected from flower buds with length of $7-9 \mathrm{~cm}(n=2)$, and striped bars those for mature pollen grains $(n=3)$. Standard errors are shown by straight lines at the tops of bars.

constituents in these lipid classes were 16:0. Molecular species composition of $\mathrm{PC}, \mathrm{PE}$ and PI of mature pollen are shown in Table IV, indicating that the molecular compositions among these phospholipids are similar. According to these results, molecular species are classified into two groups; the first group including peaks 6 and 8 is $16: 0 /(18: 2,18: 3)$, and the second group, including peaks 1,3 , and $4(18: 2,18: 3) /(18: 2,18: 3)$.

\section{Changes in molecular species of $P C$ during development of pollen}

Molecular species of PC isolated from immature and mature pollens are shown in Fig. 2. Immature pollen grains were collected from flower buds $(7-9 \mathrm{~cm})$ 4-7 days before flowering. A characteristic change was more unsaturation of fatty acid constituents with pollen development. In the $(18: 2,18: 3) /(18: 2$, $18: 3)$ group, $18: 2 / 18: 2$ species decreased with increases in $18: 2 / 18: 3$ and $18: 3 / 18: 3$ species. In the $16: 0 /(18: 2,18: 3)$ group, $16: 0 / 18: 3$ increased with a decrease in $16: 0 /$ $18: 2$. A similar result was obtained with $\mathrm{PE}$.

\section{Discussion}

PC, PE, and PI, accounting for more than $80 \%$ of total phospholipids, were the major phospholipid classes present in L. longiflorum pollen grains as well as pollen grains of other plants. ${ }^{1-5)}$ Major fatty acid constituents were $16: 0,18: 1$, and $18: 2$ in Pinus ponderosa pollen phospholipids, ${ }^{2}$ and $16: 0$ and $18: 3$ in pollen phospholipids of tea, ${ }^{3)}$ rice, ${ }^{4)}$ and maize, ${ }^{4)}$ but Andrikopoulos et al. ${ }^{11}$ reported 16:0 as a major fatty acid constituent for phospholipids of $P$. halepensis pollen grains. In contrast $16: 0,18: 2$, and $18: 3$ were present in L. longiflorum phospholipids as major fatty acid constituents (Table I) as well as tobacco pollen phospholipids. ${ }^{5)}$ Thus, it may be concluded so far that $16: 0$ and $C_{18}$-unsaturated fatty acids are major fatty acid constituents in pollen phospholipids.

Recent development of -analytical methods for molecular species has revealed that fatty acid composition analysis of lipids alone is not sufficient to understand the physiological role of lipids since one lipid class is composed of many molecular species with different combinations of fatty acid constituents. ${ }^{6,8,9,12)}$ However little is known about phospholipids 
of pollen grains. We described the molecular species of phospholipids of $L$. longiflorum pollen grains in this report. PC, PE, and PI of $L$. longiflorum pollen grains were composed of 5 major molecular species that are a "eukaryotic" type. ${ }^{13)}$ These molecular species accounting for about $90 \%$ of total phospholipids were polyunsaturated and common among PC, PE, and PI. The molecular species of $18: 3 / 18: 3$ and $16: 0 / 18: 3$ in L. longiflorum pollen are also found in tea pollen as major species accounting for $85-90 \%{ }^{3}$ ) The other 3 molecular species were less unsaturated than the above two. We hypothesize that the molecular species of angiosperm pollen phospholipids are composed of two groups of (18:1, $18: 2,18: 3) /(18: 1,18: 2,18: 3)$ and $16: 0 /$ $(18: 1,18: 2,18: 3)$ from the results with $L$. longiflorum and tea, although the extent of unsaturation may vary with plant species. From their rather simple molecular species composition, pollen phospholipids may be biosynthesized in one site, possibly in the endoplasmic reticulum. ${ }^{13)}$ Further analysis will be required for establishment of a general concept for molecular species of pollen phospholipids.

Unsaturation of phospholipids is observed during the pollen development. This seems to relate to the long life of $L$. longiflorum pollen grains under dryness at a low temperature as well as tea pollen grains. Furter investigation on changes in molecular species of phospholipids during pollen development is now in progress.

Acknowledgment. This work was partly supported by Grants-in-Aid for Special Project Research (Nos. 62107002 and 01660001) from the Ministry of Education, Science and Culture of Japan.

\section{References}

1) N. K. Andrikopoulos, A. Siafaka-Kapadai, C. A. Demopoilos and V. M. Kapoilas, Phytochemistry, 24, 2953 (1985).

2) D. L. McIlwain and C. E. Ballou, Biochemistry, 5, 4054 (1966).

3) J. Sekiya, K. Yamashita, S. Nakagawa, Y. Shibata and A. Hatanaka, Agric. Biol. Chem., 52, 243 (1988).

4) S. Toriyama, K. Hinata, I. Nishida and N. Murata, Plant Cell Physiol., 29, 615 (1988).

5) A.-J. Dorne, R. Kappler, U. Kristen and E. Heinz, Phytochemistry, 27, 2027 (1988).

6) N. Murata, Plant Cell Physiol, 24, 81 (1983).

7) M. Kito, H. Takamura, H. Narita and R. Urade, $J$. Biochem., 98, 327 (1985).

8) L. A. Smith and G. A. Thompson, Jr., "High Performance Liquid Chromatography in Plant Sciences," ed. by H. F. Linskens and J. F. Jackson, Springer-Verlag, Berlin, 1987, pp. 149-169.

9) J. Kesselmeier and E. Heinz, Anal. Biochem., 144, 319 (1985).

10) L. B. Xiong, J. Sekiya and N. Shimose, Agric. Biol. Chem., 52, 1065 (1988).

11) C. F. Allen and P. Good, "Methods in Enzymology," Vol. 23, ed. by A. San Pietro, Academic Press, New York, 1971, pp. 523-547.

12) A. J. M. Driessen, T. Zheng, G. I. Veld, J. A. F. O. den Kamp and W. N. Konings, Biochemistry, 27, 865 (1988).

13) P. G. Roughan and C. R. Slack, Ann. Rev. Plant Physiol., 33, 97 (1982). 